\title{
Tumores Pélvicos em Mulheres na Pós-menopausa
}

\author{
Pelvic Tumors in Postmenopausal Women
}

\author{
Francisco Cyro Reis de Campos Prado Filho \\ Jurandyr Moreira de Andrade, Cláudia Lemos da Silva \\ Francisco José Cândido dos Reis, Antônio Alberto Nogueira \\ Maria Matheus de Sala, Sérgio Bighetti
}

\begin{abstract}
RESUM0
Objetivos: avaliar aspectos clínicos e ultra-sonográficos de tumores pélvicos na pósmenopausa e correlacioná-los com o diagnóstico final.

Pacientes e Métodos: foram seguidas prospectivamente 36 mulheres menopausadas com diagnóstico de tumor pélvico com avaliação clínica e ultra-sonografia endovaginal. Foi definida conduta de observação em tumores císticos anecóicos e/ou com septo fino único e volume inferior a $50 \mathrm{~cm}^{3}$. Foi realizada punção em tumores com as mesmas características e volume entre 50 e $100 \mathrm{~cm}^{3}$ e laparotomia exploradora para os demais casos. O diagnóstico final definiu dois grupos de pacientes: patologia benigna (28) e maligna (8).

Resultados: tumores císticos anecóicos com septo fino único são indicativos de benignidade ( $p=0,0091$ ), ao passo que a presença de tumor com áreas sólidas (mistos) é indicativa de malignidade ( $p=0,0024)$. Ascite correlacionou-se com tumores malignos ( $p=0,0278)$. Heterogeneidade do tumor, espessamento de cápsula, septação grosseira e vegetação prevalecem nos casos malignos mas sem significância ( $p>0,05)$. O volume tumoral é parâmetro indicativo de malignidade com mediana de $85,2 \mathrm{~cm}^{3} \mathrm{em}$ benignos e 452,5 $\mathrm{cm}^{3} \mathrm{em}$ malignos ( $p=0,0048$ ), estabelecendo-se corte em $295 \mathrm{~cm}^{3}$ (sensibilidade $=83,3 \%$ e especificidade $=85,2 \%$ ). Com estes critérios todas pacientes com tumores malignos foram submetidas à laparotomia e 11 pacientes com tumores benignos foram tratadas de forma conservadora (39,3\% dos casos benignos).

Conclusão: a conduta conservadora é adequada a mulheres com tumores pélvicos de pequeno volume, anecóicos ou com septo fino, sem ascite. A diferenciação de tumores complexos e/ ou volumosos benignos e malignos necessita de investigação complementar.
\end{abstract}

PALAVRAS-CHAVE: Câncer de ovário. Tumor pélvico. Ultra-sonografia. Climatério.

\section{Introdução}

Paralelamente ao envelhecimento da população, o câncer de ovário apresenta gradativo aumento em sua incidência e mortalidade sendo,

Departamento de Ginecologia e Obstetrícia da Faculdade de Medicina de Ribeirão Preto da Universidade de São Paulo Correspondência:

Jurandyr Moreira de Andrade

Departamento de Ginecologia e Obstetrícia FMRP-USP

Av. Bandeirantes, $3900-8^{\circ}$ andar

14049-900 - Ribeirão Preto - SP entre as mulheres de países industrializados, o sexto câncer mais freqüente e a quarta causa de óbito decorrente de neoplasias ${ }^{17}$.

Contrariamente ao câncer de mama que está sendo diagnosticado cada vez mais precocemente na América do Norte e Europa Ocidental, com maioria nos estádios iniciais I e IIa, o câncer de ovário ainda é diagnosticado em fases avançadas em $67 \%$ dos casos (estádios III e IV) ${ }^{17}$.

A busca de metodologia capaz de detectar precocemente o carcinoma ovariano evoluiu de práticas clínicas, como a síndrome dos ovários 
palpáveis na pós-menopausa ${ }^{3}$, para a determinação de fatores de risco individual e familiar ${ }^{1,17} \mathrm{e}$ posteriormente para o uso de métodos laboratoriais e imagenológicos ${ }^{1,7,8,18,21,23}$.

Excetuando-se os tumores de células germinativas $^{1,15}$ e os de malignidade limítrofe ${ }^{2}$, a maioria dos tumores malignos de ovário ocorrem na pós-menopausa, seja o câncer primário ${ }^{7,8,23,24,25}$ ou metastático ${ }^{10}$, porém apesar do aumento da incidência de câncer neste grupo, ainda há predominio de patologias benignas ${ }^{11,13,20,23,25}$.

A identificação de características indicativas de alto ou baixo risco de malignidade continua sendo controversa, não havendo método tão fiel quanto a exploração cirúrgica acompanhada pela anatomia patológica. Apesar de a introdução da metodologia com Doppler pulsátil e colorido ter contribuído para a diferenciação de tumores pélvicos ${ }^{11,23,24}$, muitos ainda questionam a eficácia do método para excluir malignidade em casos aparentemente benignos ou afastar benignidade em casos suspeitos ${ }^{5,6,8,19,22,25}$ quando se comparam os achados de ultra-sonografia convencional com os da dopplervelocimetria.

Com a revisão de conceitos intervencionistas irrestritos em qualquer tumoração pélvica, diferenciaram-se subgrupos (por vezes numerosos) de pacientes que se beneficiam com condutas nãocirúrgicas ${ }^{4,9,14,16}$ ou, quando estas são indicadas, de intervenção por via laparoscópica.

Definir critérios diagnósticos clínicos e morfológicos ultra-sonográficos dos tumores pélvicos para se definir a conduta, confrontandoos com o diagnóstico final, é o objetivo deste estudo.

\section{Pacientes e Métodos}

Foram avaliadas prospectivamente 159 mulheres atendidas no Setor de Oncologia Ginecológica do Departamento de Ginecologia e Obstetrícia do Hospital das Clínicas da Faculdade de Medicina de Ribeirão Preto, no período de 01 de janeiro de 1994 a 30 de junho de 1995, com diagnóstico de tumor pélvico confirmado pela ultrasonografia. Destes casos, o grupo final deste estudo foi constituído por 36 pacientes $(22,64 \%)$ que se encontravam na pós-menopausa, definida como ausência de menstruação por período igual ou maior a 12 meses e idade acima de 40 anos, ou ainda idade acima de 50 anos para mulheres histerectomizadas previamente (1 caso).

As pacientes foram avaliadas clinicamente e submetidas a ultra-sonografia endovaginal com equipamento ATL Ultramark 4, com sonda setorial na freqüência de 5,0 MHz. Foram excluídas da casuística casos com patologia tumoral diagnosticada primariamente como uterina.

Foram avaliados aspectos ultra-sonográficos morfológicos das lesões, que foram classificadas como císticas, mistas (área sólida ocupando mais de $25 \%$ do tumor) e sólidas, além da ecogenicidade da lesão. Foram também pesquisadas a presença de septação (considerada espessa acima de $3 \mathrm{~mm}$ ), espessamento da cápsula (quando maior que $3 \mathrm{~mm}$ ), vegetação intracística e ascite (líquido livre na cavidade estendendo-se além da pelve verdadeira). O volume tumoral foi calculado pelo produto dos três eixos da lesão, que foi multiplicado pela constante 0,52 .

Foi indicada conduta clínica expectante para as pacientes com tumores císticos simples (ou com septo fino isolado) de conteúdo anecóico homogêneo e volume menor que $50 \mathrm{~cm}^{3}$ ( 5 casos). Cistos com as mesmas características, porém com volume entre 50 e $100 \mathrm{~cm}^{3}$, foram puncionados sob visualização ultra-sonográfica (6 casos), sendo o aspirado enviado para análise citológica. Pacientes de ambos os grupos foram seguidas com reavaliação clínica e ultra-sonográfica trimestral até 12 meses após o diagnóstico inicial, sendo indicada exploração cirúrgica quando houvesse alteração nas características do cisto (grupo de observação) ou recidiva do cisto (grupo de punção).

Pacientes foram encaminhadas diretamente para a exploração cirúrgica se apresentassem cistos simples com volume maior que $100 \mathrm{~cm}^{3}$, cistos complexos (heterogêneos, multiloculados ou apresentando septo grosseiro, cápsula espessa, vegetação ou ascite) e tumores mistos ou sólidos.

A avaliação cirúrgica incluiu a inspeção da cavidade, lavado peritoneal pré e pós-operatório, biópsia peritoneal das goteiras parieto-cólicas e análise histológica da peça no intra-operatório. O procedimento foi definido pela histologia e pelas condições cirúrgicas, sendo os casos com tumores benignos resolvidos individualmente. As pacientes com tumores malignos foram submetidas a histerectomia total (HTA), salpingoooforectomia bilateral (SOB), omentectomia e linfadenectomia seletiva.

A análise estatística utilizou o indice de assimetria em valor absoluto (IA $<1$ ) e coeficiente de Pearson $(<30 \%)$ na definição de simetria das amostras (paramétricas ou não). As amostras qualitativas foram avaliadas pelo teste exato de Fisher, ao passo que as quantitativas paramétricas pelo teste $t$ de Student e as não-paramétricas pelo teste de Mann-Whitney. Determinação de sensibilidade e especificidade foi feita com curva ROC. Em todos os testes utilizou-se intervalo de confiança de $95 \%$. 
Baseado no diagnóstico final foram definidos dois grupos de pacientes: grupo I com 28 casos de patologia benigna $(77,8 \%)$ e grupo II com 8 casos de patologia maligna $(22,2 \%)$.

\section{Resultados}

A média da idade das pacientes do grupo I foi 59,3 anos ao passo que a das do grupo II foi 63,6 anos, porém esta diferença não é significativa $(p=0,2425)$. O tempo decorrido entre a menopausa e a ocorrência do tumor (Figura 1) foi de 19,5 anos no grupo II e 6,5 anos no grupo I ( $p=0,0331$ ).

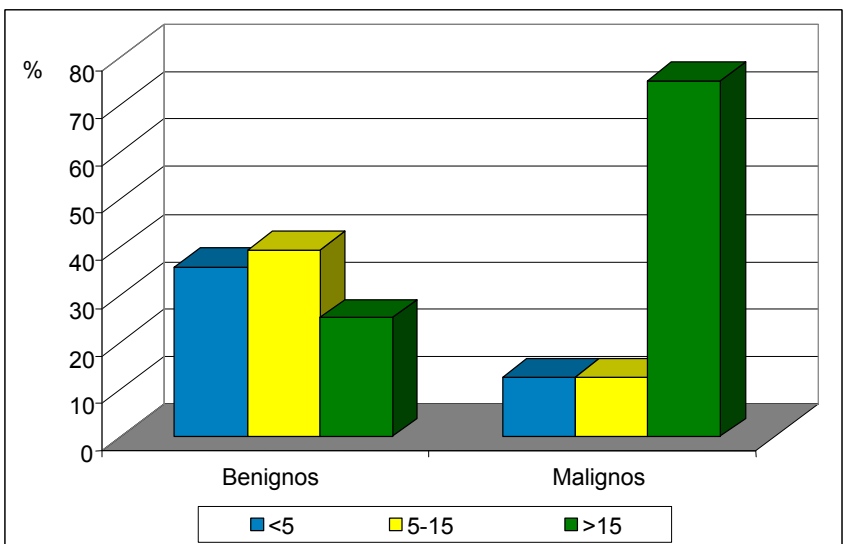

Figura 1 - Tempo decorrido de menopausa em anos, entre pacientes com tumores benignos $(n=28)$ e malignos $(n=8)$ :

Estabelecendo-se uma curva ROC, determinou-se um ponto de corte do tempo de menopausa em 16 anos, com sensibilidade de $75 \%$ e especificidade de $72,2 \%$.

O número de gestações prévias foi semelhante entre os grupos I e II, com medianas de 4 e 3,5 respectivamente $(p=0,6072)$.

Queixas clínicas foram relatadas em 8 mulheres do grupo I (28,5\%), ao passo que no grupo II foram 6 os casos (75\%) com manifestações clínicas associadas (Tabela 1), com $p=0,0361$ (teste exato de Fisher). Em 18 casos do grupo I (64,2\%) e 2 do grupo II (25\%) a tumoração pélvica era assintomática, tendo sido diagnosticada pela ultrasonografia. Em duas pacientes do grupo I $(7,1 \%)$ o tumor foi achado casual de exame de rotina, sem manifestação clínica.

O aspecto ultra-sonográfico predominante foi de cisto simples no grupo I (64,3\%), ao passo que nos malignos foi misto em 6 casos $(75 \%)$, sendo esta a única característica isolada capaz de diferenciar os grupos I e II, p = 0,0024 em teste exato de Fisher (Figura 2).

Tabela 1 - Presença de manifestações clínicas iniciais associadas a tumoração pélvica em 28 pacientes com tumores benignos e 8 com tumores malignos.

\begin{tabular}{llccc}
\hline & \multicolumn{2}{c}{ Grupo I } & \multicolumn{2}{c}{ Grupo II } \\
\cline { 2 - 5 } \multicolumn{1}{c}{ Queixa clínica } & $\mathbf{n}$ & \% & n & \% \\
\hline Dor no baixo ventre & 5 & 17,8 & 1 & 12,5 \\
Distensão abdominal & - & - & 3 & 37,5 \\
Tumoração abdominal & 1 & 3,6 & 2 & 25 \\
Dor + tumoração & 1 & 3,6 & - & - \\
Sangramento vaginal & 1 & 3,6 & - & - \\
Achado de exame clínico & 2 & 7,1 & - & - \\
Achado de ultra-som de rotina & 18 & 64,2 & 2 & 25 \\
\hline
\end{tabular}

Grupo I = tumores benignos

Grupo II = tumores malignos

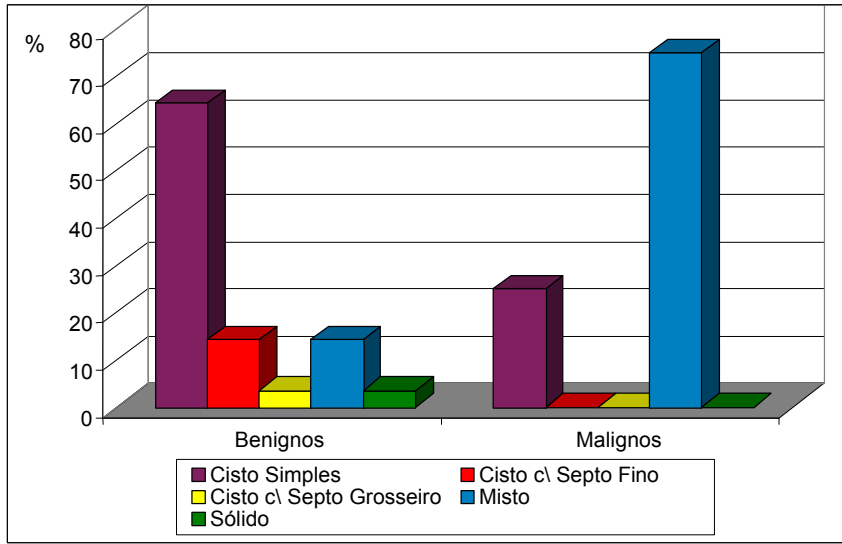

Figura 2 - Aspecto ultra-sonográfico predominante do tumor anexial em porcentagem dos tumores benignos $(n=28)$ e malignos $(n=8)$.

Agrupando-se as características ultrasonográficas em três grupos, definiram-se lesões de baixo risco (cistos simples ou com septos finos), risco moderado (cistos com septos grosseiros) e alto risco (mistas e sólidas), com resultados expressivos nos dois extremos, sendo as lesões de baixo risco presentes em $78,5 \%$ das patologias benignas $(\mathrm{p}=0,0091)$ e as de alto risco em $75,0 \%$ das malignas $(p=0,0049)$. 
A avaliação isolada de aspectos especiais à ultra-sonografia não revelou diferença entre a proporção dos grupos I e II para tumor heterogêneo e/ou multiloculado ( $\mathrm{p}=0,2169)$, cápsula espessada $(p=0,3048)$, septação grosseira $(p=0,0615)$ e vegetações intracísticas $(0,0615)$. A presença de ascite foi mais prevalente no grupo II ( $p=0,0278)$.

$\mathrm{O}$ volume tumoral (Figura 3) apresentou mediana de $85,2 \mathrm{~cm}^{3}$ no grupo I e de $452,5 \mathrm{~cm}^{3}$ no grupo II $(\mathrm{p}=0,0048)$.

Estabelecendo-se uma curva ROC para a determinação de sensibilidade e especificidade, obtiveram 2 pontos de corte distintos, com distanciamento máximo da linha média (Tabela 2).

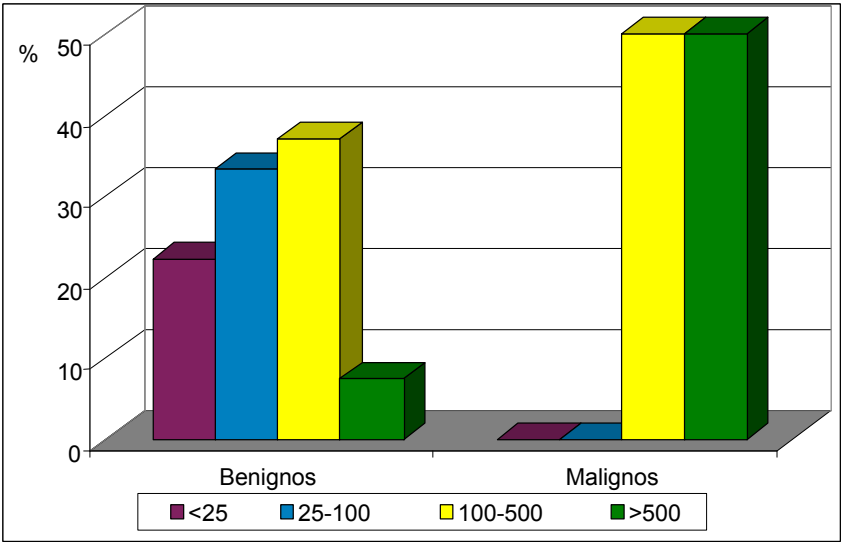

Figura 3 - Volume tumoral à ultra-sonografia em $\mathrm{cm}^{3}$ em tumores benignos $(n=28) \mathrm{e}$ malignos $(n=8)$

Tabela 2 - Sensibilidade e especificidade para o diagnóstico de malignidade em tumores pélvicos relacionados ao valor de corte para o volume tumoral.

\begin{tabular}{lccc}
\hline & Volume $\left(\mathrm{cm}^{3}\right)$ & Sensibilidade & Especificidade \\
\hline Ponto A & 295 & $83,3 \%$ & $85,2 \%$ \\
Ponto B & 180 & $100 \%$ & $70,4 \%$ \\
\hline
\end{tabular}

O tratamento das pacientes do grupo I foi baseado nos achados do exame ultra-sonográfico. Dos 5 casos de observação clínica (17,85\%), 4 (80\%) apresentaram remissão completa da lesão após 1 ano de seguimento ( 3 já na primeira reavaliação trimestral e 1 após 9 meses), ao passo que uma paciente permaneceu com a lesão inicial após 1 ano, mas com redução do volume, inalterado após 2 anos. As seis pacientes submetidas a punção guiada por ultra-sonografia $(21,42 \%)$ apresentaram citologia negativa do aspirado, com 4 delas sem recidiva $(66,66 \%)$ após doze meses e 2 casos de aspirado mucóide encaminhados à laparotomia (um cisto simples e um teratoma maduro cístico). Todas as pacientes seguidas clinicamente e as quatro puncionadas que não apresentaram recidiva receberam o diagnóstico final de cisto simples de ovário. Foram diretamente encaminhadas à cirurgia 16 pacientes deste grupo.

No grupo II, 7 pacientes foram operadas, ao passo que uma foi tratada clinicamente com quimioterapia e radioterapia por ausência de condição cirúrgica (carcinoma de colo uterino metastático para ovário). Foram realizadas 5 cirurgias completas (HTA + SOB + omentectomia + exploração da cavidade).

O diagnóstico final incluiu, dentre as patologias benignas, 21 casos $(75 \%)$ de patologias ovarianas, 3 casos $(10,8 \%)$ de patologias dos anexos, 2 casos de patologias primárias do útero $(7,1 \%)$ e 2 casos de patologia não-ginecológica $(7,1 \%)$ (Tabela 3).

Tabela 3 - Diagnóstico final das tumorações pélvicas benignas $(\mathrm{N}=28)$

\begin{tabular}{lr} 
Origem & \\
\hline Ovariana (21) & 10 \\
cisto simples & 06 \\
cistoadenoma seroso & 02 \\
$\qquad$ mucinoso & 02 \\
teratoma cístico maduro & 01 \\
tecoma & 0
\end{tabular}

Anexial (3)

torção anexial

hidrossalpinge

abscesso do ligamento largo

Uterina (2)

mioma degenerado

hidrometra

Não-ginecológica (2)

pseudocisto peritonial de retenção

hérnia gigante de disco L4-L5

01

Entre os tumores malignos, 5 casos eram primários de ovário, 4 dos quais epiteliais (1 de malignidade limítrofe) e 1 de cordões sexuais 
(granulosa), 2 metastáticos (1 de colo uterino e 1 de endométrio) e 1 de origem indeterminada (carcinomatose peritoneal difusa com ovários sem neoplasia).

Agrupando-se os dados obtidos (Tabela 4), 12 pacientes do grupo I $(42,85 \%)$ apresentaram-se com menos de 16 anos de menopausa e tumores císticos com volume abaixo de $295 \mathrm{~cm}^{3}$, ao passo que 4 pacientes do grupo II (50\%) apresentaram características inversas.

Tabela 4 - Correlação entre o tempo de menopausa, o aspecto do tumor ao ultra-som, o volume tumoral e o diagnóstico final em tumores benignos $(\mathrm{N}=28)$ e malignos $(\mathrm{N}=8)$.

\begin{tabular}{ccccc}
\hline & & & Benigno & Maligno \\
\hline Tempo de & Aspecto ultra- & Volume & & \\
menopausa & sonográfico & tumoral & & \\
$<16$ anos & cisto simples & $<295 \mathrm{~cm}^{3}$ & 12 & 0 \\
$>16$ anos & misto & $>295 \mathrm{~cm}^{3}$ & 0 & 4 \\
\hline
\end{tabular}

$p=0,0005$, teste exato de Fisher.

\section{Discussão}

A incidência de doenças como hipertensão, diabetes e outras é freqüente na mulher menopausada, aumentando consideravelmente o risco cirúrgico. O ideal é evitar a exposição a este risco inerente à cirurgia de tumores pélvicos, limitando-se as indicações de laparotomia exploradora a casos selecionados ${ }^{16,20}$, ao invés de realizá-la de forma indiscriminada a todo e qualquer aumento ovariano como se propunha no passado ${ }^{3}$.

Definir os aspectos diagnósticos que devem ser considerados na diferenciação de tumores benignos e malignos ainda permanece controverso, a despeito de vários estudos que empregam as mais diversas abordagens imagenológicas e laboratoriais, cada qual reivindicando superioridade na predição de malignidade quando comparada aos outros métodos ${ }^{7,8,11,18,24}$.

Inicialmente a clínica pode fornecer indicativos de malignidade, em especial o tempo de menopausa, verificado nesta casuística com corte em 16 anos. A presença de sintomatologia associada também foi capaz de diferenciar tumores benignos e malignos, sendo os primeiros principalmente detectados por exame ultrasonográfico e avaliação clínica em pacientes assintomáticas, ao passo que os malignos acompanharam-se de sintomatologia em $75 \%$ das pacientes. Há, contudo, contestações a estes achados, sendo descritos estudos sem qualquer diferença tanto no tempo de menopausa, quanto na presença de sintomas associados ${ }^{11,20}$.

Isoladamente a ultra-sonografia morfológica endovaginal apresenta grande variedade de caracteristicas de tumores pélvicos, com melhor ou pior capacidade de diferenciar tumores benignos e malignos. Em nossa casuística os melhores determinantes de benignidade foram o caráter cístico anecóico sem septos ou com apenas septo fino único, aspecto aceito como indicador de baixo risco $^{5,9,16,23,25}$, indicador inclusive de conduta clínica conservadora (observação ou punção) ${ }^{4,6,9,16}$. Devese entretanto ressaltar que 2 cistos associados a malignidade apresentavam esta característica, apesar de um deles apresentar ascite associada, que por si é indicativo de malignidade ${ }^{18}$.

A presença de tumoração mista (áreas císticas e sólidas) foi indicativo importante de malignidade $(p=0,0024)$, sendo mais freqüentemente associada a câncer que as tumorações sólidas ${ }^{13,25}$. As massas sólidas muitas vezes são expressão de patologias benignas, em especial as de origem uterina nãodetectadas inicialmente pela ultra-sonografia como contíguas ao útero ${ }^{21,25}$.

Outros parâmetros classicamente associados com malignidade, como heterogeneidade, septação grosseira, cápsula espessada e vegetação ${ }^{2,12,24}$, foram prevalentes entre os malignos, mas a diferença não chegou ao limiar de significância estatística, provavelmente em função da discrepância de tamanho entre as amostras benigna e maligna.

O volume tumoral foi maior nos casos malignos $(p=0,0048)$, com dois pontos de corte em 180 e $295 \mathrm{~cm}^{3}$. Considerou-se ideal o corte em $295 \mathrm{~cm}^{3}$, pois tanto a sensibilidade quanto a especificidade estão acima de $80 \%$, ao passo que com o corte em $180 \mathrm{~cm}^{3}$ é possivel detectar todos casos malignos, em detrimento de uma queda na especificidade. Coincidentemente, no estudo de Tailor et al. ${ }^{24}$ empregou-se corte em $180 \mathrm{~cm}^{3}$, mas com sensibilidade e especificidade de 73 e $75 \%$, respectivamente, em casuística de 67 casos $(52$ benignos e 15 malignos), proporção semelhante à deste trabalho.

A utilização de volume tumoral ao invés do maior diâmetro parece ser menos sujeita a variações entre observadores distintos, porém os valores definidos em protocolo devem-se relacionar diretamente com diâmetro clássico de 3,5 a $5 \mathrm{~cm}$ em cistos simples, indicativo de conduta conservadora $^{9,16 \cdot 18,20,23}$. Calculando-se o volume a partir destes diâmetros, ter-se-iam volumes entre 25 e $65 \mathrm{~cm}^{3}$, ao passo que o diâmetro de $10 \mathrm{~cm}$ 
utilizado por alguns como forte indicativo de risco ${ }^{2,20}$ corresponde a volume de $500 \mathrm{~cm}^{3}$ aproximadamente, valor próximo a mediana de $452,5 \mathrm{~cm}^{3}$ observada na presente casuística. Devese ressaltar entretanto que apesar de não haver neste estudo nenhum tumor maligno com volume inferior a $180 \mathrm{~cm}^{3}$, na literatura é freqüente o relato de pequenos tumores ovarianos de $3 \mathrm{~cm}$ ou até menos, mesmo antes do advento do ultrasom $^{3,9,16,20,23,24,25}$.

A terapêutica instituída demonstrou ser adequada ao critério de inclusão pois as pacientes tratadas conservadoramente evoluíram de forma favorável, mesmo incluindo-se as 2 pacientes puncionadas que foram encaminhadas à cirurgia, com diagnóstico final de patologia benigna. A necessidade de avaliações subseqüentes ao uso de condutas conservadoras é largamente recomendada na literatura ${ }^{4,9,16}$ e nossa conduta de reavaliações trimestrais mostrou-se adequada ao seguimento destas pacientes.

A realização de punção guiada pela ultrasonografia é metodologia eficaz, porém passivel de duas importantes ressalvas. Primeiramente há o risco de disseminação cavitária de células de tumores malignos ${ }^{14}$, apesar de ser questionável a capacidade destas células de se implantarem no peritônio. Secundariamente há ainda o risco de falso-negativo na citologia do conteúdo do cisto aspirado ${ }^{14,19}$.

O diagnóstico final das pacientes avaliadas demonstra a propriedade do termo tumor pélvico em detrimento de massa anexial, pois este último infere patologias de natureza ginecológica dos constituintes dos anexos uterinos e por vezes são encontradas patologias de origem uterina, que na ultra-sonografia aparentam independência do útero, sendo comum a inclusão de alguns casos de miomas uterinos ${ }^{8}$, especialmente se degenerados $^{9}$, e patologias extraginecológicas de natureza intestinal, como divertículos ${ }^{7}$ e tumores benignos $^{20}$, além de carcinomas de bexiga, cólon, linfomas e outras patologias menos comuns, de curiosidade descritiva apenas, como a hérnia de disco presente nesta casuística.

A dopplervelocimetria aprimorou a avaliação dos tumores, por descrever aspectos de fluxo intra e peritumoral, bem como por quantificar este fluxo ${ }^{11}$. As avaliações iniciais apontavam alta sensibilidade e especificidade do método na diferenciação de tumores ovarianos benignos e malignos ${ }^{11}$. Ensaios posteriores revelaram, entretanto, que a dopplervelocimetria era capaz de diagnosticar grande parte dos tumores malignos, mas muitas vezes não os diferenciava dos benignos, apresentando sensibilidade de 51 a $100 \%$ e especificidade de 46 a 96\% em função dos valores de corte adotados para os índices de resistência ou pulsatilidade ${ }^{5,6,22,25}$. Quando se estabelecem valores de 0,8 a 0,6 a sensibilidade aumenta consideravelmente, porém a especificidade cai abaixo de $50 \%$, ocorrendo o inverso se este índice passa a $0,45,6,22$. Alguns autores relatam valores mais altos de sensibilidade e especificidade, acima de $80 \%$, porém não encontram diferença quando comparados com a ultra-sonografia morfológica convencional ${ }^{8,18,19,23,25}$.

A associação de métodos ultra-sonográficos (morfológico e dopplervelocimetria) à quantificação sérica de CA 125 parece melhorar o resultado final, com taxa de diferenciação de até $100 \%$ quando os 3 exames são concordantes ${ }^{8,18}$, porém isoladamente o CA 125 se mostra o menos sensivel dos métodos na predição de malignidade $8,18,19,23$.

Idealmente a avaliação de um tumor pélvico deveria incluir, além do exame clínico, a ultrasonografia endovaginal morfológica com estudo com Doppler colorido, e pulsátil, associando-se ainda a tomografia helicoidal e ressonância magnética e talvez marcadores tumorais variados como CA 125 , antígeno carcinoembrionário, alfa-fetoproteína, ß - HCG, CA 19-9, CA 15-3 e tantos outros que ainda possam ser descritos. Há que se salientar, entretanto, que a maioria dos serviços médicos não dispõe de equipamento e muito menos de recursos capazes de custear tal investigação. Grande parte possui (quando muito) um equipamento de ultrasonografia convencional sem recurso de Doppler, realidade esta presente inclusive em paises industrializados como atestam Davies et al. ${ }^{7}$ no Reino Unido. Considerando-se estes argumentos, conclui-se que a ultra-sonografia endovaginal é a metodologia ideal para a avaliação de tumores pélvicos, sendo mais eficaz que outros métodos isoladamente.

Concluímos que a avaliação morfológica de tumores pélvicos em mulheres na pós-menopausa (primeiras duas décadas) pode auxiliar até $40 \%$ das pacientes com tumores benignos apresentados como cistos simples anecóicos ou com septação fina isolada e volume inferior a $295 \mathrm{~cm}^{3}$. Nestes casos, condutas conservadoras são cabíveis e seguras, desde que se mantenha seguimento e reavaliação periódicos, sendo preferencial a simples observação clínica em detrimento da punção guiada. Ao contrário, tumores mistos com volume superior a $295 \mathrm{~cm}^{3}$ em mulheres com 16 ou mais anos de menopausa devem ser avaliados de forma ativa com espécime histológico obtido em laparotomia exploradora com anatomopatologia intra-operatória e complementação cirúrgica quando necessário, diante do elevado risco de tumores malignos neste grupo de pacientes. 


\section{SUMMARY}

Purpose: to evaluate clinical and ultrasonic findings in patients with pelvic tumors at postmenopause and to correlate them with the final diagnosis.

Patients and Methods: thirty-six postmenopausal women with pelvic tumor diagnosis were prospectively evaluated through clinical examination and endovaginal ultrasonography. Clinical follow-up with no surgical procedures was indicated for anechoic cystic tumors with or without thin unique septation and volume under $50 \mathrm{~cm}^{3}$. Needle aspiration was indicated for tumors with the same aspect, and volume of 50 to $100 \mathrm{~cm}^{3}$, whereas exploratory laparotomy was performed in the remaining patients. Diagnosis defined two groups of patients: benign (28) and malignant (8) pathologies.

Results: anechoic cystic tumor with or without a thin septum indicates benignity ( $p=0.0091)$. Tumors with solid areas indicate malignancy $(p=0.0024)$. Ascites correlates with malignancy $(p=0.0278)$. Heterogeneity, thick capsule, thick septa, and papillary projections predominated in malignancies but without no statistical significance $(p>0,05)$. Tumor volume indicates malignancy, with a median of $85.2 \mathrm{~cm}^{3}$ in benign tumors and $452.5 \mathrm{~cm}^{3}$ in malignancies ( $\left.p=0.0048\right)$, with a cutoff at $295 \mathrm{~cm}^{3}$ (sensitivity $=83.3 \%$ and specificity $=$ $85.2 \%$ ). Following this protocol, all malignancies were submitted to surgery and 11 benign tumor patients were treated with a conservative protocol (39.3\% of all benign patients). Conclusion: conservative management is an adequate protocol for women with anechoic pelvic tumors with low volume, with or without single thin septum and without ascites. Differentiation between benign and malignant of complex and/or high volume tumors requires complementary investigation.

KEY WORDS: Pelvic neoplasm. Ultrasonography. Menopause.

\section{Referências}

1. Altchek A. Symptoms and signs of ovarian tumors, syndromes and susceptibility to ovarian cancer. In: Altchek A \& Deligdisch L, editores. Diagnosis and Management of Ovarian Disorders. New York: Igaku-Shoin; 1996. p. 231-49.

2. Andrade JM, Llorach-Velludo MA, Cosiski Marana HR, Murta EFC, Couto ALF, Bighetti S. Tumores de ovário de malignidade limitrofe. Rev Bras Ginecol Obstet 1996; 18:665-74.

3. Barber HR, Graber EA. The postmenopausal palpable ovary syndrome. Obstet Gynecol 1971; 38:321-3.

4. Bortoletto CCR, Gonçalves WJ, Nicolau SM, Baracat EC, Lima GR. Aspectos atuais no tratamento dos tumores císticos de ovário. Femina 1997; 25: 171-8.
5. Bromley B, Goodman H, Benacerraf BR. Comparison between sonographic morphology and Doppler waveform for the diagnosis of ovarian malignancy. Obstet Gynecol 1994; 83:434-7.

6. Brown DL, Frates MC, Laing FC, DiSalvo DN, Doubilet PM, Benson CB, et al. Ovarian masses: can benign and malignant lesions be differentiated with color and pulsed Doppler US? Radiology 1994; 190:333-6.

7. Davies AP, Jacobs I, Woolas R, Fish A, Oram D. The adnexal mass: benign or malignant? Evaluation of a risk of malignancy index. $\mathrm{Br} \mathrm{J}$ Obstet Gynaecol 1993; 100:927-31.

8. Franchi M, Beretta P, Ghezzi F, Zanaboni F, Goddi A, Salvatore S. Diagnosis of pelvic masses with transabdominal color Doppler, CA 125 and ultrasonography. Acta Obstet Gynecol Scand 1995; 74:734-9.

9. Goldstein SR, Subramanyam B, Snyder JR, Beller U, Raghavendra N, Beckman M. The postmenopausal cystic adnexal mass: the potential role of ultrasound in conservative management. Obstet Gynecol 1989; 73:8-10.

10. Guedes Neto EP, Napoli G, Montegia P, Rebhahn JS, Fontes PO. Tumores metastáticos de ovário. Rev Bras Cancerol 1993; 39:221-4.

11. Kurjak A, Schulman H, Sosic A, Zalud I, Shalan H. Transvaginal ultrasound, color flow, and Doppler waveform of the postmenopausal adnexal mass. Obstet Gynecol 1992; 80:917-21.

12. Lerner JP, Timor-Tritsch IE. Morphological evaluation of the ovary using transvaginal sonography. In: Kurjak A, editor. Ultrasound and the Ovary. New York: Parthenon; 1994. p. 115-28.

13. Luxman D, Bergman A, Sagi J, David MP. The postmenopausal adnexal mass: correlation between ultrasonic and pathologic findings. Obstet Gynecol 1991; 77:726-8.

14. Matthes AC, Moreira de Andrade J, Bighetti S. Selection of criteria for the treatment of ovarian cysts on the bases of ultrasound and cytology. Gynecol Obstet Invest 1996; 42:244-8.

15. Murta EFC, Andrade JM, Freitas MMS, Bighetti S. Neoplasias malignas não-epiteliais do ovário: estudo retrospectivo de 49 casos. Acta Oncol Bras $1995 ; 15: 147-52$

16. Pardo J, Kaplan B, Levavi H, Nitke S, Mashiach R, Neri A. Conservative management of postmenopausal pelvic echo-free findings preliminary results. Maturitas 1996; 23:279-83.

17. Piver MS, DiCioccio RA. The etiology of ovarian cancer. In: Altchek A \& Deligdisch L, editores. Diagnosis and Management of Ovarian Disorders. New York: Igaku-Shoin; 1996. p. 77-91.

18. Predanic M, Vlahos N, Pennisi JA, Moukhatar M, Aleem FA. Color and pulsed Doppler sonography, gray-scale imaging, and serum CA 125 in the 
assessment of adnexal disease. Obstet Gynecol 1996; 88:283-8.

19. Reis FJC. Diagnóstico diferencial de massas anexiais císticas: avaliação de métodos pré e pósintervenção. Ribeirão Preto. Dissertação de Mestrado. Faculdade de Medicina de Ribeirão Preto. Universidade de São Paulo, 1997.

20. Rulin MC, Preston AL. Adnexal masses in postmenopausal women. Obstet Gynecol 1987; 70:578-81.

21. Scoutt LM, McCarthy SM, Lange R, Bourque A, Schwartz PE. MR evaluation of clinically suspected adnexal masses. J Comput Assist Tomogr 1994; 18:609-18.

22. Stein SM, Laifer-Narin S, Johnson MB, Roman LD, Muderspach LI, Tyszka JM, et al. Differentiation of benign and malignant adnexal masses: relative value of gray-scale, color Doppler, and spectral Doppler sonography. Am J Roenterol 1995; 164:381-6.

23. Strigini FAL, Gadducci A, Del Bravo B, Ferdeghini M, Genazzani AR. Differential diagnosis of adnexal masses with transvaginal sonography, color flow imaging, and serum CA 125 assay in pre- and postmenopausal women. Gynecol Oncol 1996; 61:68-72.

24. Tailor A, Jurkovic D, Bourne TH, Collins WP, Campbell S. Sonographic prediction of malignancy in adnexal masses using multiparametric logistic regression analysis. Ultrasound Obstet Gynecol 1997; 10:41-7.

25. Valentin L, Sladkevicius P, Marsal K. Limited contribution of Doppler velocimetry to the differential diagnosis of extrauterine pelvic tumors. Obstet Gynecol 1994; 83:425-33.

\section{COMUNICADO AOS ASSOCIADOS E LEITORES}

Para informações e consultas temos à disposição quatro endereços eletrônicos:

$$
\begin{gathered}
\text { febrasgopresidencia@alphanet.com.br } \\
\text { secretaria_executiva@febrasgo.org.br } \\
\text { publicacoes@febrasgo.org.br } \\
\text { tego_habilitacao@febrasgo.org.br }
\end{gathered}
$$

